\title{
Sublinear eigenvalue problems on compact Riemannian manifolds with applications in Emden-Fowler equations
}

\author{
by \\ Alexandru Kristály (Cluj-Napoca) \\ and ViCEnŢıU RĂDUlescu (Bucureşti and Craiova)
}

\begin{abstract}
Let $(M, g)$ be a compact Riemannian manifold without boundary, with $\operatorname{dim} M \geq 3$, and $f: \mathbb{R} \rightarrow \mathbb{R}$ a continuous function which is sublinear at infinity. By various variational approaches, existence of multiple solutions of the eigenvalue problem

$$
-\Delta_{g} \omega+\alpha(\sigma) \omega=\tilde{K}(\lambda, \sigma) f(\omega), \quad \sigma \in M, \omega \in H_{1}^{2}(M),
$$

is established for certain eigenvalues $\lambda>0$, depending on further properties of $f$ and on explicit forms of the function $\tilde{K}$. Here, $\Delta_{g}$ stands for the Laplace-Beltrami operator on $(M, g)$, and $\alpha, \tilde{K}$ are smooth positive functions. These multiplicity results are then applied to solve Emden-Fowler equations which involve sublinear terms at infinity.
\end{abstract}

1. Introduction and statement of main results. Let us consider the following parametrized Emden-Fowler (or Lane-Emden) equation:

$(E F)_{\lambda} \quad-\Delta u=\lambda|x|^{s-2} K(x /|x|) f\left(|x|^{-s} u\right), \quad x \in \mathbb{R}^{d+1} \backslash\{0\}$,

where $f: \mathbb{R} \rightarrow \mathbb{R}$ is a continuous function, $K$ is smooth on the $d$-dimensional unit sphere $S^{d}, d \geq 3, s \in \mathbb{R}$, and $\lambda>0$ is a parameter. The equation $(E F)_{\lambda}$ has been extensively studied in the pure superlinear case, i.e., when $f$ has the form $f(t)=|t|^{p-1} t, p>1$ (see Cotsiolis-Iliopoulos [3], Vázquez-Véron [9]). In these papers, the authors obtained existence and multiplicity of solutions for $(E F)_{\lambda}$, applying either minimization or minimax methods. Note that in the pure superlinear case the presence of the parameter $\lambda>0$ is not relevant due to the rescaling technique. One of the purposes of the present paper is to guarantee multiple solutions of $(E F)_{\lambda}$ for certain $\lambda>0$ when the nonlinearity $f: \mathbb{R} \rightarrow \mathbb{R}$ is

(a) not necessarily of pure power type, and

(b) sublinear at infinity (see (1.2) below).

2000 Mathematics Subject Classification: 58J05, 35J60.

Key words and phrases: Emden-Fowler equation, sublinear eigenvalue problem, multiple solutions. 
The solutions of $(E F)_{\lambda}$ are being sought in the particular form

$$
u(x)=u(|x|, x /|x|)=u(r, \sigma)=r^{s} \omega(\sigma) .
$$

Here, $(r, \sigma) \in(0, \infty) \times S^{d}$ are the spherical coordinates in $\mathbb{R}^{d+1} \backslash\{0\}$. This type of transformation is also used by Bidaut-Véron and Véron [2], where the asymptotics of a special form of $(E F)_{\lambda}$ has been studied. By means of $(1.1)$, the equation $(E F)_{\lambda}$ reduces to

$$
-\Delta_{g_{0}} \omega+s(1-s-d) \omega=\lambda K(\sigma) f(\omega), \quad \sigma \in S^{d},
$$

where $\Delta_{g_{0}}$ denotes the Laplace-Beltrami operator on $\left(S^{d}, g_{0}\right)$ and $g_{0}$ is the canonical metric induced from $\mathbb{R}^{d+1}$.

Note in particular that when $s=-d / 2$ or $s=-d / 2+1$, and $f(t)=$ $|t|^{4 /(d-2)} t$, the existence of a smooth solution $\omega>0$ of $(1.1)_{\lambda}$ can be viewed as an affirmative answer to the famous Yamabe problem on $S^{d}$ (see also the Nirenberg problem); for these topics we refer the reader to Aubin [1], Cotsiolis-Iliopoulos [4], Hebey [5], and references therein. In these cases the right hand side of $(1.1)_{\lambda}$ involves the critical Sobolev exponent.

As we pointed out before, our aim is to study $(E F)_{\lambda}$ (specially, problem $\left.(1.1)_{\lambda}\right)$ in the sublinear case. Since $1-d<s<0$ implies the coercivity of the operator $\omega \mapsto-\Delta_{g_{0}} \omega+s(1-s-d) \omega$, the form of $(1.1)_{\lambda}$ motivates the study of the following general eigenvalue problem, denoted by $(P)_{\lambda}$, which constitutes the main objective of our paper:

Find $\lambda \in(0, \infty)$ and $\omega \in H_{1}^{2}(M)$ such that

$$
-\Delta_{g} \omega+\alpha(\sigma) \omega=\tilde{K}(\lambda, \sigma) f(\omega), \quad \sigma \in M,
$$

where we assume

$\left(A_{1}\right)(M, g)$ is a smooth compact d-dimensional Riemannian manifold without boundary, $d \geq 3$;

$\left(A_{2}\right) \alpha \in C^{\infty}(M)$ and $\tilde{K} \in C^{\infty}((0, \infty) \times M)$ are positive functions;

$\left(f_{1}\right) f: \mathbb{R} \rightarrow \mathbb{R}$ is locally Hölder continuous and sublinear at infinity, i.e.,

$$
\lim _{|t| \rightarrow \infty} \frac{f(t)}{t}=0 .
$$

A typical case when (1.2) holds is

$\left(f_{1}^{q, c}\right)$ There exist $q \in(0,1)$ and $c>0$ such that $|f(t)| \leq c|t|^{q}$ for every $t \in \mathbb{R}$.

We simply say that $\omega_{\lambda} \in H_{1}^{2}(M)$ is a solution of $(P)_{\lambda}$ if $\omega=\omega_{\lambda}$ satisfies $(1.1)_{\lambda}$. Above, $\Delta_{g}$ is the Laplace-Beltrami operator on $(M, g)$; its expression in local coordinates is $\Delta_{g} \omega=g^{i j}\left(\partial_{i j} \omega-\Gamma_{i j}^{k} \partial_{k} \omega\right)$. $H_{1}^{2}(M)$ is the usual Sobolev space on $M$, endowed with its natural norm $\|\cdot\|_{H_{1}^{2}}$ (see [5] or Section 2 for details). 
The presence of the parameter $\lambda>0$ in $(P)_{\lambda}$ is indispensable. Indeed, if we consider a sublinear function at infinity which is, in addition, uniformly Lipschitz (with Lipschitz constant $L>0$ ), and $\tilde{K}(\lambda, \sigma)=\lambda K(\sigma)$, with $K \in$ $C^{\infty}(M)$ positive, one can prove that for $0<\lambda<(1 / L)\left(\min _{M} \alpha / \max _{M} K\right)$ $=: \lambda_{L}$ we have only the $\omega=\omega_{\lambda}=0$ solution of $(P)_{\lambda}$, as the standard contraction principle on the Hilbert space $H_{1}^{2}(M)$ shows. For a concrete example, let us consider the function $f(t)=\ln \left(1+t^{2}\right)$ and assume that $K(\sigma) / \alpha(\sigma)=$ const $=\mu_{0} \in(0, \infty)$. Then, for every $0<\lambda<\min _{M} \alpha / \mu_{0} \max _{M} \alpha$, problem $(P)_{\lambda}$ has only the trivial solution; however, when $\lambda>5 / 4 \mu_{0}$, problem $(P)_{\lambda}$ has three distinct constant solutions which are precisely the fixed points of the function $t \mapsto \lambda \mu_{0} \ln \left(1+t^{2}\right)$. Note that one of the solutions is the trivial one.

In the generic case, for a fixed $\lambda>0$, the function $\omega_{\lambda}(\sigma)=c \in \mathbb{R}$ is a solution of $(P)_{\lambda}$ if and only if $\alpha(\sigma) c=\tilde{K}(\lambda, \sigma) f(c)$ for a.e. $\sigma \in M$. In particular, when $\omega_{\lambda}(\sigma)=c \neq 0$, the function $\sigma \mapsto \tilde{K}(\lambda, \sigma) / \alpha(\sigma)$ is constant; let us denote this value by $\mu_{\lambda}>0$. Thus, nonzero constant solutions of $(P)_{\lambda}$ appear as fixed points of the function $t \mapsto \mu_{\lambda} f(t)$.

In order to obtain multiple solutions of $(P)_{\lambda}$ not only in the case when $\sigma \mapsto \tilde{K}(\lambda, \sigma) / \alpha(\sigma)$ is constant for certain $\lambda>0$, we will use variational arguments; weak solutions of $(P)_{\lambda}$ will be found as critical points of the energy functional associated with $(P)_{\lambda}$ (see Section 2). Due to $\left(f_{1}\right)$, these elements are actually classical solutions of $(P)_{\lambda}$.

Our first result concerns the case when $f: \mathbb{R} \rightarrow \mathbb{R}$ satisfies

$\left(f_{2}\right) \lim _{t \rightarrow 0} f(t) / t=0$.

Before stating this result, let us note that the usual norm on the space $L^{p}(M)$ will be denoted by $\|\cdot\|_{p}, p \in[1, \infty]$.

Theorem 1.1. Assume that $f: \mathbb{R} \rightarrow \mathbb{R}$ satisfies $\left(f_{1}\right),\left(f_{2}\right)$ and $\sup _{t \in \mathbb{R}} F(t)$ $>0$, where $F(t)=\int_{0}^{t} f(\tau) d \tau$. Assume also that $\left(A_{1}\right)$ and $\left(A_{2}\right)$ are satisfied with $\tilde{K}(\lambda, \sigma)=\lambda K(\sigma)$, and $K \in C^{\infty}(M)$ is positive. Then there exists $\tilde{\lambda}>0$ such that for every $\lambda>\tilde{\lambda}$ problem $(P)_{\lambda}$ has at least two distinct, nontrivial solutions.

REMARK 1.1. As we mentioned above, when the nonlinearity $f$ is a uniformly Lipschitz function (with Lipschitz constant $L>0$ ), we have extra information on the eigenvalues:

(a) problem $(P)_{\lambda}$ has only the trivial solution whenever $\lambda \in\left(0, \lambda_{L}\right)$;

(b) problem $(P)_{\lambda}$ has at least two nontrivial solutions whenever $\lambda>\tilde{\lambda}$.

Moreover, the proof of Theorem 1.1 shows that the number $\tilde{\lambda}$ is less than or equal to the value 


$$
\lambda^{*}:=\frac{1}{2} \frac{\|\alpha\|_{1}}{\|K\|_{1}}\left(\max _{t \neq 0} \frac{F(t)}{t^{2}}\right)^{-1} .
$$

Consequently, Theorem 1.1 is valid for any $\lambda>\lambda^{*}$ which can be easily computed.

A direct consequence of Theorem 1.1 applied for $(1.1)_{\lambda}$ is the following

Theorem 1.2. Assume that $1-d<s<0$. Let $f: \mathbb{R} \rightarrow \mathbb{R}$ be a function as in Theorem 1.1 and $K \in C^{\infty}\left(S^{d}\right)$ positive. Then there exists $\tilde{\lambda}>0$ such that for every $\lambda>\tilde{\lambda}$ problem $(E F)_{\lambda}$ has at least two distinct, nontrivial solutions.

In order to obtain a new kind of multiplicity result concerning $(P)_{\lambda}$ (specially, $(1.1)_{\lambda}$ and $\left.(E F)_{\lambda}\right)$, we require:

$\left(f_{3}\right)$ There exists $\mu_{0} \in(0, \infty)$ such that the set of all global minima of the function $t \mapsto \tilde{F}_{\mu_{0}}(t):=\frac{1}{2} t^{2}-\mu_{0} F(t)$ has at least $m \geq 2$ connected components.

Note that $\left(f_{3}\right)$ implies that the function $t \mapsto \tilde{F}_{\mu_{0}}(t)$ has at least $m-1$ local maxima. Thus, the function $t \mapsto \mu_{0} f(t)$ has at least $2 m-1$ fixed points. In particular, if for some $\lambda>0$ one has $\tilde{K}(\lambda, \sigma) / \alpha(\sigma)=\mu_{0}$ for every $\sigma \in M$, then problem $(P)_{\lambda}$ has at least $2 m-1 \geq 3$ constant solutions. On the other hand, the following general result can be shown.

TheOREM 1.3. Let $f: \mathbb{R} \rightarrow \mathbb{R}$ be a function which satisfies $\left(f_{1}\right)$ and $\left(f_{3}\right)$. Assume that $\left(A_{1}\right)$ and $\left(A_{2}\right)$ are satisfied with $\tilde{K}(\lambda, \sigma)=\lambda K(\sigma)+\mu_{0} \alpha(\sigma)$, and $K \in C^{\infty}(M)$ is positive. Then

(a) for every $\eta>\max \left\{0,\|\alpha\|_{1} \min _{t \in \mathbb{R}} \tilde{F}_{\mu_{0}}(t)\right\}$ there exists $\tilde{\lambda}_{\eta}>0$ such that for every $\lambda \in\left(0, \tilde{\lambda}_{\eta}\right)$ problem $(P)_{\lambda}$ has at least $m+1$ solutions $\omega_{\lambda}^{1, \eta}, \ldots, \omega_{\lambda}^{m+1, \eta} \in H_{1}^{2}(M)$

(b) if $\left(f_{1}^{q, c}\right)$ holds then for each $\lambda \in\left(0, \tilde{\lambda}_{\eta}\right)$ there is a set $I_{\lambda} \subset\{1, \ldots$, $m+1\}$ with $\operatorname{card}\left(I_{\lambda}\right)=m$ such that

$$
\left\|\omega_{\lambda}^{i, \eta}\right\|_{H_{1}^{2}}<\frac{t_{\eta, q, c}}{\min \left\{1, \min _{M} \alpha^{1 / 2}\right\}}, \quad i \in I_{\lambda},
$$

where $t_{\eta, q, c}>0$ is the greatest solution of the equation

$$
\frac{1}{2} t^{2}-\frac{\mu_{0} c\|\alpha\|_{1}^{(1-q) / 2}}{q+1} t^{q+1}-\eta=0, \quad t>0 .
$$

A consequence of Theorem 1.3 in the context of $(E F)_{\lambda}$ reads as follows.

TheOREM 1.4. Assume that $1-d<s<0$. Let $f: \mathbb{R} \rightarrow \mathbb{R}$ be a function as in Theorem 1.3 and $K \in C^{\infty}\left(S^{d}\right)$ be a positive function. Then there exists $\lambda_{0}>0$ such that for every $\lambda \in\left(0, \lambda_{0}\right)$ the problem 


$$
-\Delta u=|x|^{s-2}\left[\lambda K(x /|x|)+\mu_{0} s(1-s-d)\right] f\left(|x|^{-s} u\right), \quad x \in \mathbb{R}^{d+1} \backslash\{0\},
$$

has at least $m+1$ solutions.

The proof of Theorem 1.1 is based on the paper of Pucci-Serrin [6], while that of Theorem 1.3 relies on a recent abstract critical point theorem of Ricceri (see [7] and [8]).

Examples. (a) Let $f(t)=\ln \left(1+t^{2}\right)$. Then Theorems 1.1-1.2 apply.

(b) Let $f: \mathbb{R} \rightarrow \mathbb{R}$ be defined by $f(t)=\min \left\{t_{+}-\sin \left(\pi t_{+}\right), 2(m-1)\right\}$ where $m \in \mathbb{N} \backslash\{1\}$ is fixed and $t_{+}=\max \{t, 0\}$. Clearly, $\left(f_{1}\right)$ is satisfied, while for $\mu_{0}=1$, the assumption $\left(f_{3}\right)$ also holds. Indeed, the function $t \mapsto \tilde{F}_{1}(t)$ has precisely $m$ global minima: $0,2, \ldots, 2(m-1)$. Moreover, $\min _{t \in \mathbb{R}} \tilde{F}_{1}(t)=0$. Therefore, one can apply Theorems 1.3 and 1.4.

In the next section we recall some basic facts on Sobolev spaces defined on compact Riemannian manifolds. In Section 3 we prove Theorems 1.1 and 1.2 while in the last section we deal with the proofs of Theorems 1.3 and 1.4.

2. Preliminaries. Let $(M, g)$ be a smooth compact $d$-dimensional Riemannian manifold without boundary, $d \geq 3$, and let $\alpha \in C^{\infty}(M)$ be a positive function. For every $\omega \in C^{\infty}(M)$, set

$$
\|\omega\|_{H_{\alpha}^{2}}^{2}=\int_{M}\langle\nabla \omega, \nabla \omega\rangle d \sigma_{g}+\int_{M} \alpha(\sigma)\langle\omega, \omega\rangle d \sigma_{g},
$$

where $\langle\cdot, \cdot\rangle$ is the inner product on covariant tensor fields associated to $g$, $\nabla \omega$ is the covariant derivative of $\omega$, and $d \sigma_{g}$ is the Riemannian measure. The Sobolev space $H_{\alpha}^{2}(M)$ is defined as the completion of $C^{\infty}(M)$ with respect to the norm $\|\cdot\|_{H_{\alpha}^{2}}$. Clearly, $H_{\alpha}^{2}(M)$ is a Hilbert space endowed with the inner product

$$
\left\langle\omega_{1}, \omega_{2}\right\rangle_{H_{\alpha}^{2}}=\int_{M}\left\langle\nabla \omega_{1}, \nabla \omega_{2}\right\rangle d \sigma_{g}+\int_{M} \alpha(\sigma)\left\langle\omega_{1}, \omega_{2}\right\rangle d \sigma_{g}, \quad \omega_{1}, \omega_{2} \in H_{\alpha}^{2}(M) .
$$

Since $\alpha$ is positive, the norm $\|\cdot\|_{H_{\alpha}^{2}}$ is equivalent to the standard norm $\|\cdot\|_{H_{1}^{2}}$; actually, the latter is just $\|\cdot\|_{H_{\alpha}^{2}}$ with $\alpha=1$. Moreover, we have

$$
\min \left\{1, \min _{M} \alpha^{1 / 2}\right\}\|\omega\|_{H_{1}^{2}} \leq\|\omega\|_{H_{\alpha}^{2}} \leq \max \left\{1,\|\alpha\|_{\infty}^{1 / 2}\right\}\|\omega\|_{H_{1}^{2}}
$$

for $\omega \in H_{\alpha}^{2}(M)$. Note that $H_{\alpha}^{2}(M)$ is compactly embedded in $L^{p}(M)$ for every $p \in[1,2 d /(d-2))$; the Sobolev embedding constant will be denoted by $S_{p}>0$.

Let $\lambda>0$. The energy functional $\mathcal{E}_{\lambda}: H_{1}^{2}(M) \rightarrow \mathbb{R}$ associated with problem $(P)_{\lambda}$ is

$$
\mathcal{E}_{\lambda}(\omega)=\frac{1}{2}\|\omega\|_{H_{\alpha}^{2}}^{2}-\int_{M} \tilde{K}(\lambda, \sigma) F(\omega(\sigma)) d \sigma_{g},
$$


where $F(t)=\int_{0}^{t} f(\tau) d \tau$. Due to our initial assumptions $\left(A_{1}\right),\left(A_{2}\right)$ and $\left(f_{1}\right)$, the functional $\mathcal{E}_{\lambda}$ is well-defined, it belongs to $C^{1}\left(H_{1}^{2}(M), \mathbb{R}\right)$, and its critical points are precisely the weak (so classical) solutions of problem $(P)_{\lambda}$. By $\left(f_{1}\right)$, for every $\varepsilon>0$ sufficiently small there is $c(\varepsilon)>0$ such that $|f(t)| \leq \varepsilon|t|+c(\varepsilon)$ for every $t \in \mathbb{R}$. Consequently, for every $\omega \in H_{1}^{2}(M)$, we have

$$
\mathcal{E}_{\lambda}(\omega) \geq \frac{1}{2}\left(1-\varepsilon\|\tilde{K}(\lambda, \cdot)\|_{\infty} S_{2}^{2}\right)\|\omega\|_{H_{\alpha}^{2}}^{2}-c(\varepsilon)\|\tilde{K}(\lambda, \cdot)\|_{\infty} S_{1}\|\omega\|_{H_{\alpha}^{2}} .
$$

Therefore, the functional $\mathcal{E}_{\lambda}$ is coercive and bounded from below on $H_{1}^{2}(M)$. Moreover, it satisfies the standard Palais-Smale condition (see Zeidler [10, Example 38.25]).

3. Proof of Theorems 1.1 and 1.2. In this section we assume the hypotheses of Theorem 1.1 hold. We define $\mathcal{N}, \mathcal{F}: H_{1}^{2}(M) \rightarrow \mathbb{R}$ by

$$
\mathcal{N}(\omega)=\frac{1}{2}\|\omega\|_{H_{\alpha}^{2}}^{2} \quad \text { and } \quad \mathcal{F}(\omega)=\int_{M} K(\sigma) F(\omega(\sigma)) d \sigma_{g}, \quad \omega \in H_{1}^{2}(M) .
$$

Proposition 3.1. $\lim _{\varrho \rightarrow 0^{+}} \sup \{\mathcal{F}(\omega) / \mathcal{N}(\omega): 0<\mathcal{N}(\omega)<\varrho\}=0$.

Proof. Due to $\left(f_{2}\right)$, for small $\varepsilon>0$ there exists $\delta(\varepsilon)>0$ such that $|f(t)|<\varepsilon\left(2\|K\|_{\infty} S_{2}^{2}\right)^{-1}|t|$ for every $|t|<\delta(\varepsilon)$. On account of $\left(f_{1}\right)$, one may fix $1<\nu<(d+2) /(d-2)$ and $c(\varepsilon)>0$ such that $|f(t)|<c(\varepsilon)|t|^{\nu}$ for every $|t| \geq \delta(\varepsilon)$. Combining these two facts, after an integration, we obtain

$$
|F(t)| \leq \varepsilon\left(4\|K\|_{\infty} S_{2}^{2}\right)^{-1} t^{2}+c(\varepsilon)(\nu+1)^{-1}|t|^{\nu+1} \quad \text { for every } t \in \mathbb{R} .
$$

Fix a $\varrho>0$ and any $\omega \in H_{\alpha}^{2}(M)$ with $\mathcal{N}(\omega)<\varrho$. By the above estimate,

$$
\begin{aligned}
\mathcal{F}(\omega) & \leq \frac{\varepsilon}{4}\|\omega\|_{H_{\alpha}^{2}}^{2}+\frac{c(\varepsilon)}{\nu+1}\|K\|_{\infty} S_{\nu+1}^{\nu+1}\|\omega\|_{H_{\alpha}^{2}}^{\nu+1} \\
& <\frac{\varepsilon}{4}\|\omega\|_{H_{\alpha}^{2}}^{2}+\frac{c(\varepsilon)}{\nu+1}\|K\|_{\infty} S_{\nu+1}^{\nu+1}(2 \varrho)^{(\nu-1) / 2}\|\omega\|_{H_{\alpha}^{2}}^{2} \\
& =\left(\varepsilon / 4+c^{\prime}(\varepsilon) \varrho^{(\nu-1) / 2}\right)\|\omega\|_{H_{\alpha}^{2}}^{2} .
\end{aligned}
$$

Thus there exists $\varrho(\varepsilon)>0$ such that for every $0<\varrho<\varrho(\varepsilon)$, we have

$$
0 \leq \sup \{\mathcal{F}(\omega) / \mathcal{N}(\omega): 0<\mathcal{N}(\omega)<\varrho\} \leq \varepsilon / 2+2 c^{\prime}(\varepsilon) \varrho^{(\nu-1) / 2}<\varepsilon,
$$

which completes the proof.

Proof of Theorem 1.1. Let us define

$$
\tilde{\lambda}=\inf \{\mathcal{N}(\omega) / \mathcal{F}(\omega): \mathcal{F}(\omega)>0\} .
$$

Fix $\lambda>\tilde{\lambda}$. On one hand, there exists $\omega_{\lambda}^{0} \in H_{1}^{2}(M)$ such that $\mathcal{F}\left(\omega_{\lambda}^{0}\right)>0$ and $\lambda>\mathcal{N}\left(\omega_{\lambda}^{0}\right) / \mathcal{F}\left(\omega_{\lambda}^{0}\right)$. On account of (2.2) and (3.1), we have

$$
\mathcal{E}_{\lambda}\left(\omega_{\lambda}^{0}\right)=\mathcal{N}\left(\omega_{\lambda}^{0}\right)-\lambda \mathcal{F}\left(\omega_{\lambda}^{0}\right)<0 .
$$


On the other hand, due to Proposition 3.1, there exists $\delta>0$ such that for all $\varrho \in(0, \delta)$ one has

$$
\sup \{\mathcal{F}(\omega) / \mathcal{N}(\omega): 0<\mathcal{N}(\omega)<\varrho\}<1 / \lambda .
$$

In other words, for every $\omega \in H_{1}^{2}(M) \backslash\{0\}$ satisfying $\mathcal{N}(\omega)<\varrho$ we have $\mathcal{F}(\omega) / \mathcal{N}(\omega)<1 / \lambda$ and hence

$$
\mathcal{E}_{\lambda}(\omega)=\mathcal{N}(\omega)-\lambda \mathcal{F}(\omega)>0=\mathcal{E}_{\lambda}(0) .
$$

Consequently, $0 \in H_{1}^{2}(M)$ is a local minimum point for $\mathcal{E}_{\lambda}$, but not a global one, in view of (3.2). Since the functional $\mathcal{E}_{\lambda}$ is bounded from below on $H_{1}^{2}(M)$ and it satisfies the standard Palais-Smale condition, the global minimum of $\mathcal{E}_{\lambda}$ is achieved. Applying [6, Theorems 1 and 4], we obtain a third critical point of $\mathcal{E}_{\lambda}$ which is not 0 . This concludes our proof.

Proof of Theorem 1.2. Let us choose $(M, g)=\left(S^{d}, g_{0}\right)$, and $\alpha(\sigma)=$ $s(1-s-d)$ for every $\sigma \in S^{d}$ in Theorem 1.1. Thus, for every $\lambda>\tilde{\lambda}$, problem $(1.1)_{\lambda}$ has at least two distinct, nontrivial solutions $\omega_{\lambda}^{1}, \omega_{\lambda}^{2} \in H_{1}^{2}\left(S^{d}\right)$. On account of (1.1), the elements $u_{\lambda}^{i}(x)=|x|^{s} \omega_{\lambda}^{i}(x /|x|), i \in\{1,2\}$, are solutions of $(E F)_{\lambda}$.

REMARK 3.1. For every $k \in\{1, \ldots, d-2\}$, let $G_{k}=O(k+1) \times O(d-k) \subset$ $O(d+1)$. The action of the group $G_{k}$ on $H_{1}^{2}\left(S^{d}\right)$ is defined by $g \omega(\sigma)=$ $\omega\left(g^{-1} \sigma\right)$ for every $g \in G_{k}, \omega \in H_{1}^{2}\left(S^{d}\right), \sigma \in S^{d}$. Assume that $K$ in $(1.1)_{\lambda}$ is constant. One can prove that the energy functional $\mathcal{E}_{\lambda}: H_{1}^{2}\left(S^{d}\right) \rightarrow \mathbb{R}$ is $G_{k}$-invariant for every $k \in\{1, \ldots, d-2\}$, i.e., $\mathcal{E}_{\lambda}(g \omega)=\mathcal{E}_{\lambda}(\omega)$ for every $g \in G_{k}$ and $\omega \in H_{1}^{2}\left(S^{d}\right)$. Now, let

$$
H_{G_{k}}\left(S^{d}\right)=\left\{\omega \in H_{1}^{2}\left(S^{d}\right): g \omega=\omega \text { for every } g \in G_{k}\right\}
$$

be the fixed point space of $H_{1}^{2}\left(S^{d}\right)$ under the action of $G_{k}$. Let $\mathcal{E}_{\lambda}^{G_{k}}$ be the restriction of the functional $\mathcal{E}_{\lambda}$ to the space $H_{G_{k}}\left(S^{d}\right)$. Now, we follow the same arguments as in Theorem 1.1 with $\mathcal{E}_{\lambda}^{G_{k}}$ and $H_{G_{k}}\left(S^{d}\right)$ instead of $\mathcal{E}_{\lambda}$ and $H_{1}^{2}\left(S^{d}\right)$, respectively. Therefore, for every $\lambda>\tilde{\lambda}$ and $k \in\{1, \ldots, d-2\}$ we can guarantee the existence of at least two different, nontrivial critical points of $\mathcal{E}_{\lambda}^{G_{k}}$ belonging to $H_{G_{k}}\left(S^{d}\right)$. On the other hand, the principle of symmetric criticality of Palais implies that every critical point of $\mathcal{E}_{\lambda}^{G_{k}}$ will also be a critical point of $\mathcal{E}_{\lambda}$, thus a solution of $(1.1)_{\lambda}$. If such a solution is not constant, say $\omega_{\lambda, k} \in H_{G_{k}}\left(S^{d}\right)$, it cannot belong to $H_{G_{l}}\left(S^{d}\right)$ whenever $l \neq k$. Indeed, when $k \neq l$, the group generated topologically by $G_{k}$ and $G_{l}$ acts transitively on $S^{d}$, that is, the orbit of every element from $S^{d}$ under that group is the whole sphere $S^{d}$. Consequently, $H_{G_{k}}\left(S^{d}\right) \cap H_{G_{l}}\left(S^{d}\right)$ contains only the a.e. constant functions defined on $S^{d}$. Therefore, a nonconstant solution of $(1.1)_{\lambda}$ which belongs to $H_{G_{k}}\left(S^{d}\right)$ will not appear in $H_{G_{l}}\left(S^{d}\right)$, $l \neq k$. In this way, the number of solutions of $(1.1)_{\lambda}$ and $(E F)_{\lambda}$ can increase. 
4. Proof of Theorems 1.3 and 1.4. We assume the hypotheses of Theorem 1.3 hold. Using the notation from the previous section (see (3.1)), we define the functional $\mathcal{N}_{\mu_{0}}: H_{1}^{2}(M) \rightarrow \mathbb{R}$ by

$$
\mathcal{N}_{\mu_{0}}(\omega)=\mathcal{N}(\omega)-\mu_{0} \int_{M} \alpha(\sigma) F(\omega(\sigma)) d \sigma_{g}, \quad \omega \in H_{1}^{2}(M) .
$$

Proposition 4.1. The set of all global minima of the functional $\mathcal{N}_{\mu_{0}}$ has at least $m$ connected components in the weak topology on $H_{1}^{2}(M)$.

Proof. First, for every $\omega \in H_{1}^{2}(M)$ we have

$$
\begin{aligned}
\mathcal{N}_{\mu_{0}}(\omega) & =\frac{1}{2}\|\omega\|_{H_{\alpha}^{2}}^{2}-\mu_{0} \int_{M} \alpha(\sigma) F(\omega(\sigma)) d \sigma_{g} \\
& =\frac{1}{2} \int_{M}|\nabla \omega|^{2} d \sigma_{g}+\int_{M} \alpha(\sigma) \tilde{F}_{\mu_{0}}(\omega(\sigma)) d \sigma_{g} \\
& \geq\|\alpha\|_{1} \inf _{t \in \mathbb{R}} \tilde{F}_{\mu_{0}}(t) .
\end{aligned}
$$

Moreover, if we consider $\omega(\sigma)=\omega_{\tilde{t}}(\sigma)=\tilde{t}$ for a.e. $\sigma \in M$, where $\tilde{t} \in \mathbb{R}$ is a minimum point of the function $t \mapsto \tilde{F}_{\mu_{0}}(t)$, then we have equality in the previous estimate. Thus,

$$
\inf _{\omega \in H_{1}^{2}(M)} \mathcal{N}_{\mu_{0}}(\omega)=\|\alpha\|_{1} \inf _{t \in \mathbb{R}} \tilde{F}_{\mu_{0}}(t) .
$$

Moreover, if $\omega \in H_{1}^{2}(M)$ is not a constant function, then $|\nabla \omega|^{2}=g^{i j} \partial_{i} \omega \partial_{j} \omega$ $>0$ on a positive measure set in $M$. In this case,

$$
\mathcal{N}_{\mu_{0}}(\omega)=\frac{1}{2} \int_{M}|\nabla \omega|^{2} d \sigma_{g}+\int_{M} \alpha(\sigma) \tilde{F}_{\mu_{0}}(\omega(\sigma)) d \sigma_{g}>\|\alpha\|_{1} \inf _{t \in \mathbb{R}} \tilde{F}_{\mu_{0}}(t) .
$$

Consequently, there is a one-to-one correspondence between the sets

$$
\operatorname{Min}\left(\mathcal{N}_{\mu_{0}}\right)=\left\{\omega \in H_{1}^{2}(M): \mathcal{N}_{\mu_{0}}(\omega)=\inf _{\omega \in H_{1}^{2}(M)} \mathcal{N}_{\mu_{0}}(\omega)\right\}
$$

and

$$
\operatorname{Min}\left(\tilde{F}_{\mu_{0}}\right)=\left\{t \in \mathbb{R}: \tilde{F}_{\mu_{0}}(t)=\inf _{t \in \mathbb{R}} \tilde{F}_{\mu_{0}}(t)\right\} .
$$

Indeed, let $\theta$ be the function that associates to every $t \in \mathbb{R}$ the equivalence class of those functions which are a.e. equal to $t$ on the whole $M$. Then $\theta: \operatorname{Min}\left(\tilde{F}_{\mu_{0}}\right) \rightarrow \operatorname{Min}\left(\mathcal{N}_{\mu_{0}}\right)$ is actually a homeomorphism, where $\operatorname{Min}\left(\mathcal{N}_{\mu_{0}}\right)$ is considered with the relativization of the weak topology on $H_{1}^{2}(M)$. On account of the hypothesis $\left(f_{3}\right)$, the set $\operatorname{Min}\left(\tilde{F}_{\mu_{0}}\right)$ has at least $m \geq 2$ connected components. Therefore, the same is true for the set $\operatorname{Min}\left(\mathcal{N}_{\mu_{0}}\right)$, which completes the proof.

Since $\tilde{K}(\lambda, \sigma)=\lambda K(\sigma)+\mu_{0} \alpha(\sigma)$, the energy functional associated to $(P)_{\lambda}$ has the form $\mathcal{E}_{\lambda}=\mathcal{N}_{\mu_{0}}-\lambda \mathcal{F}$, where $\mathcal{F}$ comes from (3.1). In order 
to prove Theorem 1.3, we recall a recent critical point result of Ricceri [7, Theorem 8].

THEOREM 4.1. Let $H$ be a separable and reflexive real Banach space, and let $\mathcal{N}, \mathcal{G}: H \rightarrow \mathbb{R}$ be two sequentially weakly lower semicontinuous and continuously Gâteaux differentiable functionals, with $\mathcal{N}$ coercive. Assume that the functional $\mathcal{N}+\lambda \mathcal{G}$ satisfies the Palais-Smale condition for every $\lambda>0$ small enough and that the set of all global minima of $\mathcal{N}$ has at least $m$ connected components in the weak topology, with $m \geq 2$. Then, for every $\eta>\inf _{H} \mathcal{N}$, there exists $\bar{\lambda}>0$ such that for every $\lambda \in(0, \bar{\lambda})$, the functional $\mathcal{N}+\lambda \mathcal{G}$ has at least $m+1$ critical points, $m$ of which are in $\mathcal{N}^{-1}((-\infty, \eta))$.

Proof of Theorem 1.3. Let us choose $H=H_{1}^{2}(M), \mathcal{N}=\mathcal{N}_{\mu_{0}}$ and $\mathcal{G}=$ $-\mathcal{F}$ in Theorem 4.1. Due to Proposition 4.1 and to basic properties of the functions $\mathcal{N}_{\mu_{0}}, \mathcal{F}$, all the hypotheses of Theorem 4.1 are satisfied.

Then, for every $\left.\eta>\max \left\{0,\|\alpha\|_{1} \min _{t \in \mathbb{R}} \tilde{F}_{\mu_{0}}(t)\right\}\left(\geq \inf _{\omega \in H_{1}^{2}(M)} \mathcal{N}_{\mu_{0}}(\omega)\right\}\right)$ there is a $\tilde{\lambda}_{\eta}>0$ such that for every $\lambda \in\left(0, \tilde{\lambda}_{\eta}\right)$ the function $\mathcal{N}_{\mu_{0}}-\lambda \mathcal{F}$ has at least $m+1$ critical points; let us denote them by $\omega_{\lambda}^{1, \eta}, \ldots, \omega_{\lambda}^{m+1, \eta} \in H_{1}^{2}(M)$. Clearly, they are solutions of problem $(P)_{\lambda}$, which proves (a).

We know in addition that $m$ elements among $\omega_{\lambda}^{1, \eta}, \ldots, \omega_{\lambda}^{m+1, \eta}$ belong to the set $\mathcal{N}_{\mu_{0}}^{-1}((-\infty, \eta))$. Let $\tilde{\omega}$ be such an element, i.e.,

$$
\mathcal{N}_{\mu_{0}}(\tilde{\omega})=\frac{1}{2}\|\tilde{\omega}\|_{H_{\alpha}^{2}}^{2}-\mu_{0} \int_{M} \alpha(\sigma) F(\tilde{\omega}(\sigma)) d \sigma_{g}<\eta .
$$

Assume that $\left(f_{1}^{q, c}\right)$ holds. Then $|F(t)| \leq \frac{c}{q+1}|t|^{q+1}$ for every $t \in \mathbb{R}$. By the Hölder inequality, one has

$$
\int_{M} \alpha(\sigma)|\tilde{\omega}(\sigma)|^{q+1} d \sigma_{g} \leq\|\alpha\|_{1}^{(1-q) / 2}\|\tilde{\omega}\|_{H_{\alpha}^{2}}^{q+1} .
$$

Since $\eta>0$, the equation

$$
\frac{1}{2} t^{2}-\frac{\mu_{0} c\|\alpha\|_{1}^{(1-q) / 2}}{q+1}|t|^{q+1}-\eta=0
$$

always has a positive solution. On account of (4.1) and (4.2), the number $\|\tilde{\omega}\|_{H_{\alpha}^{2}}$ is less than the greatest solution $t_{\eta, q, c}>0$ of (4.3). It remains to apply (2.1) to prove (b).

Proof of Theorem 1.4. It follows directly by Theorem 1.3.

Acknowledgments. The authors thank the anonymous referee for his/her useful observations and comments concerning Theorem 1.1.

A. Kristály was supported by CNCSIS grant PN II, IDEI 527. V. Rădulescu was partially supported by Grant 2-CEx06-11-18/2006. 


\section{References}

[1] T. Aubin, Some Nonlinear Problems in Riemannian Geometry, Springer Monogr. Math., Springer, Berlin, 1998.

[2] M. F. Bidaut-Véron and L. Véron, Nonlinear elliptic equations on compact Riemannian manifolds and asymptotics of Emden equations, Invent. Math. 106 (1991), 489-539.

[3] A. Cotsiolis and D. Iliopoulos, Équations elliptiques non linéaires à croissance de Sobolev sur-critique, Bull. Sci. Math. 119 (1995), 419-431.

[4] - - - Équations elliptiques non linéaires sur $S^{n}$. Le problème de Nirenberg, C. R. Acad. Sci. Paris Sér. I Math. 313 (1991), 607-609.

[5] E. Hebey, Nonlinear Analysis on Manifolds: Sobolev Spaces and Inequalities, Courant Lecture Notes in Math. 5, Amer. Math. Soc., 2000.

[6] P. Pucci and J. Serrin, Extensions of the mountain pass theorem, J. Funct. Anal. 59 (1984), 185-210.

[7] B. Ricceri, Sublevel sets and global minima of coercive functionals and local minima of their perturbations, J. Nonlinear Convex Anal. 5 (2004), 157-168.

[8] - A multiplicity theorem for the Neumann problem, Proc. Amer. Math. Soc. 134 (2006), 1117-1124.

[9] J. L. Vázquez et L. Véron, Solutions positives d'équations elliptiques semi-linéaires sur des variétés riemanniennes compactes, C. R. Acad. Sci. Paris Sér. I Math. 312 (1991), 811-815.

[10] E. Zeidler, Nonlinear Functional Analysis and its Applications, Vol. III, Springer, Berlin, 1985.

Department of Economics

University of Babeş-Bolyai

400591 Cluj-Napoca, Romania

E-mail: alexandrukristaly@yahoo.com
Institute of Mathematics "Simion Stoilow" of the Romanian Academy 014700 Bucureşti, Romania and Department of Mathematics University of Craiova 200585 Craiova, Romania E-mail: vicentiu.radulescu@math.cnrs.fr http://www.inf.ucv.ro/ ${ }^{2}$ adulescu

Received April 20, 2008

Revised version September 18, 2008 